\title{
S'occuper des enfants, un truc de meufs ?
}

Par Émilie BILAND (sociologue et politiste, Sciences Po),

Julie MINOC (sociologue, université de Versailles Saint-Quentin-en-Yvelines)

et Hélène OEHMICHEN (sociologue, EHESS)

Remerciements à Sophie Lavagnelle (professeure de sciences économiques et sociales)

Aujourd'hui encore, les mères en font nettement plus que les pères à la maison. Ces inégalités domestiques prennent plusieurs formes : du temps pour réaliser les multiples tâches, de l'attention pour coordonner les activités des membres du foyer, des émotions et des sentiments pour apaiser et adoucir leur quotidien. Au boulot, c'est pareil : les femmes sont beaucoup plus nombreuses à travailler auprès d'enfants, dans les crèches, les écoles, les services sociaux. Est-ce parce qu'elles aiment ça ? Parce que ça les arrange ? Et les hommes, qu'est-ce qu'ils font, au juste? Comment changer les choses?

\section{À la maison : elles en font toujours plus !}

Les médias félicitent les «nouveaux pères », les «papas poules», qui s'impliquent auprès de leurs enfants. Mais les statistiques ont la dent dure : les enquêtes «Emploi du temps » de l'INSEE montrent qu'on est loin de l'égalité en matière de travail domestique. Chaque jour, une femme y passe en moyenne près de trois heures, contre moins de deux heures pour un homme.

Les choses ont néanmoins progressé : il y a quarante-cinq ans, les hommes ne prenaient en charge qu'un quart du travail domestique, contre un tiers aujourd'hui. Quant aux femmes, elles ont plus souvent une activité professionnelle hors du foyer, elles gagnent du temps grâce aux appareils électroménagers (merci le lave-vaisselle !), et celles qui le peuvent font appel aux services d'autres femmes pour alléger leur quotidien (on y reviendra). Cela étant, ce sont toujours elles qui s'occupent des tâches les moins appréciées. Les hommes comme les femmes préfèrent la plupart du temps jardiner plutôt que passer l'aspirateur. Mais les hommes s'investissent avant tout dans 
les activités vues comme agréables (bricolage ou jardinage) et dans celles qui impliquent des déplacements hors du foyer (les courses, que l'on peut faire en allant ou en sortant du travail). Auprès des enfants, ils prennent surtout part aux activités ludiques et éducatives, les femmes continuant de nourrir, laver, habiller, etc. Elles conservent aussi la charge mentale associée au travail domestique : ce sont elles qui font les listes et qui s'assurent que tout se déroule sans encombre: vaccinations des enfants selon le calendrier prévu, inscription à la crèche, visites de la famille et des amis, rencontres avec les professeurs, etc.

Dès les années 1970, les féministes matérialistes, comme Christine Delphy, ont souligné l'importance de ce travail reproductif, qui assure que les adultes seront en état de travailler (les repas sont prêts, le linge est repassé, etc.) et que les enfants apprennent à se conformer à cet ordre des choses par la socialisation. Elles ont aussi montré que ce travail est rendu invisible, notamment parce qu'il n'est pas rémunéré. Si les tâches domestiques étaient payées au SMIC, elles vaudraient plus de 600 milliards d'euros par an : c'est un tiers de la richesse nationale ! Dans nos sociétés capitalistes, quantifier ce travail féminin est indispensable pour reconnaître sa valeur.

Mais on ne peut bien sûr tout réduire à ces chiffres. La sociologue américaine Arlie R. Hochschild montre que les femmes assument aussi l'essentiel du travail émotionnel, qui consiste à gérer et susciter des sentiments et émotions appropriés chez les autres : apaiser la colère d'un enfant, faire en sorte que son conjoint puisse se détendre après le boulot fait partie du travail de soin (le care, en anglais) socialement construit comme féminin.

En plus, les transformations du marché du travail et des formes familiales ne favorisent pas toujours un partage plus égalitaire. La «double journée » professionnelle et familiale - accroît la charge mentale des femmes, ainsi que leur fatigue physique. D'ailleurs, elles sont bien plus nombreuses que les hommes à travailler à temps partiel et plus encore à prendre des congés parentaux après la naissance des enfants. En fait, c'est principalement sur elles que repose la «conciliation » entre vie professionnelle et vie familiale. Dans $75 \%$ des couples, 1'homme a un revenu supérieur à la femme : dès lors, le « choix » est souvent vite fait pour savoir qui freinera sa carrière pour s'occuper des enfants. La naissance d'un enfant a des conséquences négatives sur 
la carrière des femmes (elles perdent en moyenne entre 2 et $3 \%$ de salaire), tandis qu'elle est bénéfique pour celle des hommes, dont le salaire augmente d'en moyenne $3 \%$.

Le temps professionnel des hommes continue donc de prendre beaucoup de place. Ceux qui appartiennent aux classes populaires (ouvriers, camionneurs, etc.) ont souvent peu de latitude dans leur emploi du temps : quand on travaille tôt le matin ou tard le soir, ou qu'on s'absente plusieurs jours par semaine, pas facile d'aller chercher les enfants à l'école ou de s'occuper d'eux le mercredi. Ces positions différentes des femmes et des hommes sur le marché du travail, combinées au partage inégal des tâches quand ils vivent en couple, expliquent en large part pourquoi les enfants dont les parents sont séparés vivent la plupart du temps chez leur mère - même si la résidence alternée devient plus fréquente. $85 \%$ des familles monoparentales sont «dirigées » par une femme : pas de doute, dans ces cas-là, c'est bien la mère qui doit s'occuper des enfants en premier lieu.

Ce n'est pas parce que les femmes en font plus qu'elles sont toutes-puissantes. Pendant longtemps, c'est la «puissance paternelle» qui était inscrite dans le droit. Aujourd'hui, pères et mères ont a priori les mêmes droits et devoirs envers leurs enfants. Mais, dans les faits, l'ascendant masculin sur certaines tâches décisives pour le devenir des enfants n'a pas disparu. Les pères de classes populaires restent souvent attachés à la figure d'autorité qu'ils veulent incarner pour leurs enfants : "Par définition, je suis le chef de famille. Donc, j'essaie d'avoir cette image du chef de famille et de donner aux enfants un exemple d'autorité, de rigueur », dit l'un d'eux à la sociologue MarieClémence Le Pape. Les hommes de classes moyennes et supérieures sont plus prompts à tenir des discours égalitaires. Mais ils pèsent toujours de manière décisive sur la gestion du patrimoine familial (par exemple, pour décider de l'achat d'une maison ou de placements financiers). Ils jouent aussi un rôle majeur dans les stratégies scolaires (évitement de la carte scolaire, choix d'orientation) : leur temps est compté, mais ils l'utilisent pleinement pour conserver ou améliorer le statut social de leur famille, de génération en génération. 


\section{Au boulot : faire travailler les femmes pour « libérer » les mères ?}

Les femmes ont aujourd'hui une activité professionnelle dans des proportions proches de celle des hommes (70\% contre $75 \%$ ). Mais, quand les parents travaillent, qui s'occupe des enfants? Les cartes sont-elles rebattues hors de la maison?

Eh bien pas vraiment ! puisque les femmes sont largement majoritaires dans les maternités, les crèches, les écoles... L'État a certes favorisé le travail rémunéré des femmes et leur émancipation de la sphère privée en développant les possibilités de garde pour les tout-petits : crèches, assistantes maternelles, école maternelle, etc. Mais cet adjectif, «maternel », est significatif : ces métiers et ces lieux sont toujours pensés au féminin. Dans le secteur de la petite enfance, les hommes représentent moins de 1,5\% des effectifs.

Cette féminisation massive repose sur l'idéologie du maternage, selon laquelle les femmes seraient «prédisposées » à comprendre et prendre en charge les besoins des enfants. Cette idéologie remonte très loin dans l'histoire et produit ses effets dans toutes les institutions de prise en charge des enfants, dès leur naissance. Prenons l'exemple des sages-femmes (un autre mot féminin par défaut !). Pendant longtemps, il était nécessaire d'être mère pour accompagner les grossesses, les accouchements se déroulant d'ailleurs à domicile . En 1943, la loi encadrant la pratique de sage-femme disait qu'il fallait disposer d'une «connaissance interne, profonde et personnelle de la féminité ». Les femmes étaient alors considérées comme «les mieux placées pour se tenir auprès d'autres femmes, pour les préparer, les rassurer, les conseiller et les aider pendant la grossesse et l'accouchement ». Aujourd'hui, la pratique s'est médicalisée et distanciée du foyer. Depuis 1982, la profession est ouverte aux hommes. Pourtant, d'après l'ordre des sages-femmes, $97 \%$ d'entre elles sont des femmes. Les stéréotypes attachés à ces métiers éloignent de facto les hommes de ces professions, encore souvent perçues comme peu viriles et peu ambitieuses - en revanche, les anesthésistes qui installent les péridurales sont majoritairement des hommes !

Le fait que certains métiers soient principalement assurés par des femmes n'est pas un problème en soi. Ce qui l'est, ce sont les préjugés et les inégalités que la féminisation contribue à (re)produire entre les femmes et les hommes, les mères et les 
pères. La division des tâches dans la sphère privée se retrouve dans la sphère professionnelle, ce qui participe à la dévalorisation de ces activités. Non reconnues comme vraiment «professionnelles », les compétences de prise en charge des enfants sont perçues comme « simplement » relationnelles, affectives ou émotionnelles. Il n’y aurait pas forcément besoin de se former ou de les apprendre pour les avoir, puisqu'elles découleraient du supposé « instinct maternel ».

Attention cependant : ce ne sont pas n'importe quelles femmes qui occupent les professions les moins valorisées et les plus précaires auprès des enfants (assistantes maternelles ou familiales, personnels temporaires des écoles...) : ces femmes sont plus souvent issues de milieux sociaux populaires et immigrées. Les mères qui peuvent déléguer une partie du travail domestique et parental à d'autres femmes (en embauchant une nounou, par exemple) sont quant à elles plus souvent issues des classes moyennes ou supérieures et non immigrées. Cette délégation témoigne des inégalités entre les femmes selon leur classe et leur origine.

Quant aux hommes qui exercent auprès des enfants, ils occupent plus souvent que les femmes des fonctions d'autorité, d'encadrement ou de gestion. En fait, plus on monte dans la hiérarchie des métiers éducatifs ou de santé, plus les compétences attendues sont considérées comme «techniques» et exigent de longues études. À l'école, plus les enfants grandissent et apprennent des choses jugées plus « compliquées », plus il y a d'hommes pour leur faire classe. Selon les données du ministère de l'Éducation nationale, les hommes ne sont que 7 \% parmi les enseignants en maternelle, mais déjà $28 \%$ en primaire et $42 \%$ en collège et lycée. Si les mots « assistantes maternelles » ou « infirmières » sont presque toujours au féminin, on parle en revanche de «médecins » ou de «professeurs ». Cela ne signifie pas qu'il n'y a pas de femmes pédiatres ou professeures, mais que, dans l'inconscient collectif, ces métiers plus valorisés sont moins associés à des qualités considérées comme féminines.

Enfin, en raison de leur socialisation et de leur pratique familiale, mais aussi du fait des savoirs psychologiques appris durant leur formation, les professionnels de la petite enfance, de santé ou du travail social contribuent au partage inégal des tâches entre parents. Dans les crèches, si un enfant est malade et qu'il faut venir le récupérer, c'est généralement la mère qui est contactée. Les femmes doivent donc plus souvent 
«s'arranger » au travail pour pallier les imprévus. De manière consciente ou non, par des conseils réguliers, par des sollicitations ou des remontrances, ces professionnels accroissent le travail et la charge mentale des mères, tout en contribuant à ce que les pères délaissent le quotidien de leurs enfants.

\section{D'où ça vient et comment on s'en sort ?}

Certains diront que les femmes s'occupent davantage des enfants parce qu'elles aiment le faire, ou parce qu'elles sont plus douées que les hommes. Cette vision des choses repose sur l'idée de complémentarité des rôles, idée qui est en fait le produit de la socialisation : encore aujourd'hui, on n'est pas élevé de la même manière selon que l'on est considéré comme une fille ou comme un garçon. La division des tâches entre parents et entre professionnels a des conséquences sur les enfants. Ceux-ci s'habituent à ce que les femmes prennent soin d'eux, quand les hommes font figure d'autorité ou exercent des métiers plus techniques ou plus reconnus. Cette situation leur semble aller de soi.

Les institutions de la petite enfance sont en fait au début d'une longue chaîne d'institutions qui renvoient les filles et les garçons à des rôles sexués et alimentent des stéréotypes. De manière implicite ou explicite, les adultes ont des attentes différentes vis-à-vis des filles et des garçons. Les jouets amplifient la division sexuée des rôles : ceux destinées aux filles les préparent au travail de soin (coiffeuse, dînette, poupées, maîtresse...), tandis que ceux qui ciblent les garçons les encouragent à s'engager dans des compétitions, à montrer leur courage et leur force (voitures, jouets de guerre, policier...). Ainsi, les filles sont plus incitées à exprimer leurs émotions, à être patientes, attentives et serviables : elles développent par là des qualités qu'elles pourront réutiliser auprès des enfants. Par contraste, beaucoup d'adultes reprennent les garçons qui pleurent, mais tolèrent davantage leurs colères, quitte à ce que cette socialisation à la masculinité (qui est aussi un apprentissage de l'hétérosexualité) nuise à la réussite scolaire de certains d'entre eux, surtout dans les classes populaires.

Par mimétisme et apprentissage, les enfants se font donc progressivement une idée de ce pour quoi ils ou elles sont « faits » ou « faites », là où ils ou elles doivent être 
«bons » ou «bonnes » à la maison, à l'école et au travail. Cette socialisation, c'est ce qui va façonner leurs aspirations, leur avenir, leurs dispositions à exercer tel ou tel métier pour lequel ils et elles se sentiront «naturellement » compétents.

En bref, savoir s'occuper des enfants n'est pas une qualité naturellement féminine ; c'est un long travail de construction par l'ensemble des sphères de la société tout au long de la vie. À l'adolescence, les filles sont plus nombreuses que les garçons à travailler comment baby-sitters, et les parents ne s'en étonnent guère...

Pourtant, de plus en plus de femmes réclament que les hommes s'occupent plus des enfants, et que les métiers exercés par des femmes soient davantage valorisés. Alors pourquoi l'ordre social est-il si pesant et difficile à transformer? D'abord, prendre conscience des inégalités que produit la socialisation genrée ne suffit pas à s'en émanciper. Par ailleurs, l'État ne nous aide guère, même si les choses changent progressivement. Le système français d'impôts et de prestations sociales continue à décourager le travail des femmes. Par exemple, les conjoints mariés ou pacsés font le plus souvent une déclaration commune aux impôts ; pour cette raison, ils peuvent avoir intérêt dans certains cas à ce que le conjoint ou la conjointe qui gagne le moins (qui est, comme on l'a vu, plus souvent la femme) réduise, voire abandonne, son activité professionnelle.

En plus, la politique familiale continue de distinguer fortement pères et mères : le congé maternité dure seize semaines (pour le premier ou le deuxième enfant) tandis que le congé de paternité est de seulement onze jours ! Créé en 2002, il est utilisé par sept pères sur dix ; et ceux qui travaillent avec des contrats précaires ou qui ne sont pas salariés le prennent encore moins que les autres.

Très tôt, les inégalités de prise en charge s'installent : les mères s'occupent des nouveau-nés pendant que les pères sont au travail, et l'habitude risque d'être prise pour la suite. Une fois le congé maternité terminé, les solutions de garde hors du foyer sont insuffisantes : pour 100 enfants de moins de trois ans, il y a 18 places en crèche et 35 places chez une assistante maternelle, tandis que 44 enfants n'ont pas de solution de garde par des professionnels. Le plus souvent, il reste alors avec sa mère ou avec une autre femme de leur famille! 
Pour résumer, les femmes ne sont pas autant incitées que les hommes à exercer une activité professionnelle, dans la mesure où elles gagnent moins d'argent et où les exigences du marché du travail (longues journées de travail, déplacements etc.) sont, on l'a dit, problématiques quand on a la charge d'enfants.

Alors que faire, au niveau politique, social ou individuel, pour changer les choses ? Eh bien, agir sur tous ces niveaux à la fois !

D’abord, rendre le marché du travail plus compatible avec la prise en charge des enfants : pas de réunion tard le soir, possibilité de prendre en charge les enfants sur le lieu de travail (seulement $3 \%$ des entreprises disposent d'une crèche !). En 2017, une sénatrice australienne, Larissa Waters, allaitait son enfant en pleine séance parlementaire : cet acte politique visait à montrer qu'une jeune mère peut pleinement et légitimement occuper une fonction qualifiée, visible et prestigieuse. Ce symbole est puissant, mais il ne doit pas faire oublier que les femmes de classes populaires sont celles qui pâtissent le plus, aujourd'hui, du partage inégal des tâches domestiques combiné à la précarité du travail.

Les femmes ne s'occupent pas seulement des enfants; elles prennent en charge les personnes âgées dépendantes et les personnes en situation de handicap. Presque toujours, il s'agit d'emplois usants physiquement, peu rémunérés et peu considérés. La reconnaissance statutaire et financière de ces emplois est un enjeu majeur, à l'heure où les besoins en aide humaine vont croissant.

Les hommes devraient, quant à eux, être mieux considérés en tant que pères par leurs employeurs et par l'État. Ce n'est pas au moment des séparations conjugales qu'il faut s'inquiéter de leur place auprès des enfants, mais bien dès la vie commune, en les incitant à prendre des congés ou des temps partiels. Il y aurait beaucoup d'autres pistes à explorer, mais on ne peut terminer ce court texte sans mentionner la nécessité d'aller vers une éducation moins genrée. Arrêtons le bleu et le rose ! Encourageons les garçons à jouer à la poupée et les adolescentes à se former en informatique ! On a besoin de pères qui s'impliquent plus au foyer et de mères qui développent leur carrière en étant mieux rémunérées. 


\section{Morale de l'histoire}

Il n'est ni naturel ni normal que les femmes s'occupent prioritairement des enfants. Ce partage inégalitaire des tâches, à la maison et au travail, contribue à la hiérarchisation des genres, en dévalorisant les activités assumées par les femmes. Et il limite le champ des possibles pour l'ensemble des individus! La prise en charge des enfants tout comme le soutien aux personnes vulnérables sont des sujets trop cruciaux pour pâtir des stéréotypes de genre ; ils sont trop centraux pour qu'on accepte qu'ils perpétuent les inégalités entre femmes et hommes.

\section{Lexique de désenfumage}

Charge mentale : la charge mentale est un terme que l'on entend beaucoup dans les médias depuis quelque temps. En sociologie, il s'est imposé dès les années 1980 dans les recherches féministes. La sociologue Monique Haincault l'utilise alors pour désigner la prise en charge mentale, invisible et constante, que représente le fait de devoir anticiper et coordonner les tâches domestiques. Généralement dévolue aux femmes, la charge mentale consiste ainsi au fait de penser aux moindres détails de l'organisation de la vie familiale tout le temps, n'importe où, et pour chaque membre de la famille : prendre rendez-vous chez le médecin pour le petit dernier, penser à racheter des couches et du café, rappeler à son conjoint que, demain, c'est l'anniversaire de sa mère pour qu'il n'oublie pas de lui téléphoner, etc.

Dispositions : ce que la sociologie nomme les « dispositions » d'un individu, ce sont toutes les manières de faire, de dire et de penser qu'il a intériorisées au cours de son existence. Le sociologue Pierre Bourdieu a beaucoup travaillé cette idée, afin d'expliquer pourquoi un individu, dans un contexte donné, va avoir tendance à se comporter comme ceci ou comme cela, à faire tel choix plutôt que tel autre, etc. Par exemple, en offrant aux filles, dès le plus jeune âge, des poupées pour jouer, on les « dispose » plus que les garçons à s'occuper des enfants. Contrairement à ce que, dans 
le langage ordinaire, on appelle « prédispositions », les dispositions, en sociologie, sont construites socialement, c'est-à-dire qu'on ne naît pas avec, on les acquiert par le processus de socialisation.

Socialisation : la socialisation est un processus par lequel, dès la naissance et tout au long de sa vie, chaque individu apprend à se conformer aux règles et aux attentes de la vie en société. Des valeurs et des normes nous sont ainsi transmises par divers «agents socialisateurs » (famille, école, amis, travail, médias, etc.). C'est ce qui va construire notre identité sociale et faire que l'on s'intègre à la société. La socialisation primaire, qui a lieu au cours de l'enfance, est souvent considérée comme déterminante, car les premiers repères sociaux que l'on acquiert à ce moment-là marquent durablement nos manières de penser et d'agir.

Stéréotype : un stéréotype est une image ou une représentation que l'on se fait d'une personne ou d'un groupe, souvent en caricaturant certains traits de caractère, réels ou supposés. Par exemple, l'idée que les hommes sont moins sensibles que les femmes est un stéréotype. Il s'agit d'une manière simple, mais aussi souvent simpliste, de catégoriser les gens qui nous entourent et de se situer vis-à-vis d'eux. Les stéréotypes orientent ainsi notre perception du monde social et les comportements que l'on adopte à l'égard des autres. Ils sont socialement partagés, c'est-à-dire que les médias, les amis, la famille, pensent comme nous et contribuent à orienter l'idée qu'on se fait de certaines catégories de personnes.

Travail du care: la notion de care apparaît aux États-Unis au début des années 1980 sous la plume de la psychologue Carol Gilligan. Mais c'est la philosophe Joan Tronto qui, dans les années 1990, contribue à sa diffusion. Le care peut être défini comme le « souci des autres », et plus exactement le fait d'apporter une réponse concrète aux besoins des autres. En ce sens, le travail du care renvoie aux activités liées aux soins aux personnes âgées, malades, dépendantes mais s'étend plus largement à la prise en charge des enfants et personnes qui partagent notre vie quotidienne. Aux frontières du domestique, du sanitaire et du social, le travail du care comprend une dimension 
matérielle (changer une couche, faire la toilette, etc.), une dimension morale ou éthique, intégrant l'attention, la présence, l'empathie et la bienveillance accompagnant cette activité de soin donné à autrui et, enfin, une dimension politique liée à la reconnaissance de la valeur associée à ce travail relationnel, souvent exercé par des femmes.

Travail émotionnel: par ce terme, la sociologue Arlie R. Hochschild fait référence à la façon dont les individus maîtrisent ou gèrent leurs émotions et celles des autres, dans la vie quotidienne et au travail, afin de les mettre en adéquation avec les attentes de la société. Pour le dire autrement, le travail émotionnel, ce sont tous les efforts déployés par une personne pour exprimer ou afficher les émotions que lui dicte une situation sociale donnée, mais aussi ceux déployés pour susciter ou générer des émotions appropriées chez les autres.

Travail reproductif : le travail reproductif est ce qui permet de reconstituer continuellement les forces humaines pour que le travail productif puisse être assuré. Plus précisément, il désigne à la fois la reproduction biologique des travailleurs, la prise en charge et la formation des futures recrues sur le marché du travail (les enfants) et le fait que les adultes soient toujours en état de travailler (repas, habillement, soin...). Ce terme trouve son origine dans les travaux de Karl Marx et Friedrich Engels qui, à la fin du $\mathrm{XIX}^{\mathrm{e}}$ siècle, lui opposaient le «travail productif » des salariés. Pour eux, contrairement à ce dernier, le travail reproductif ne produit pas de «plus-value » (voir fiche « Le travail coûte trop cher en France ? ») et est d'ailleurs principalement cantonné à la sphère privée. Dans les années 1970, les penseuses féministes ont critiqué cette distinction : elles ont montré que le travail reproductif est loin d'être négligeable et qu'il est indispensable de le considérer comme un travail au même titre que le travail productif. Cela permet de rendre visible, d'une part, le fait que le travail se déploie à la fois dans la sphère de production et dans la sphère privée (qui n'est pas qu'un lieu de consommation !) et, d'autre part, que le travail reproductif en particulier participe d'un mode de domination qui opprime les femmes : le patriarcat. 


\section{$\underline{\text { À lire }}$}

Nadia Amrous et Françoise Borderies, «L'offre d'accueil collectif des enfants de moins de trois ans en $2015 »$, document de travail n 203, DREES, 2018

Cécile Brousse, «Travail professionnel, tâches domestiques, temps "libre": quelques déterminants sociaux de la vie quotidienne », Économie et statistique, $\mathrm{n}^{\circ} 478$ 480, 2015, p. 119-154

Clara Champagne, Ariane Pailhé et Anne Solaz, «Le temps domestique et parental des hommes et des femmes : quels facteurs d'évolutions en vingt-cinq ans ? », Économie et statistique, $\mathrm{n}^{\circ} 478-480,2015$, p. 209-242

Élise Coudin, Sophie Maillard et Maxime Tô, «Entreprises, enfants : quels rôles dans les inégalités salariales entre femmes et hommes ? », INSEE Analyses, $\mathrm{n}^{\circ} 44$, février 2019

Anne Dafflon Novelle (dir.), Filles-garçons: socialisation différenciée?, Presses universitaires de Grenoble, Grenoble, 2006

Christine Delphy, L’Ennemi principal. Économie politique du patriarcat, Syllepses, Paris, 2013

Hervé Gosselin et Carole Lépine, «Évaluation du congé de paternité », rapport de l'Inspection générale des affaires sociales, Paris, 2018

Caroline Ibos, Qui gardera nos enfants? Les nounous et les mères, Flammarion, Paris, 2012

Danièle Kergoat, Se battre, disent-elles..., La Dispute, Paris, 2012

Yvonne Knibiehler (dir.), Maternité : affaire privée, affaire publique, Bayard, Paris, 2001

Arlie R. Hochschild, Le Prix des sentiments. Au cœur du travail émotionnel, La Découverte, Paris, 2017

Le Collectif Onze, Au tribunal des couples : enquête sur des affaires familiales, Odile Jacob, Paris, 2013

Marie-Clémence Le Pape, «Être parent dans les milieux populaires : entre valeurs traditionnelles familiales et nouvelles normes éducatives », Informations sociales, $\mathrm{n}^{\circ} 154,2009$, p. 88-95 
Wilfried Lignier, Prendre. Naissance d'une pratique sociale élémentaire, Le Seuil, Paris, 2019

Marie-Cécile Naves et Vanessa Wisnia-Weill (dir.), «Lutter contre les stéréotypes filles-garçons. Un enjeu d'égalité et de mixité dès l'enfance », rapport du Commissariat général à la stratégie et à la prospective, 2014

OCDE, «Efficacité et équité du système de prélèvements et de transferts », Études économiques de l'OCDE, vol. 5, $\mathrm{n}^{\circ}$ 5, 2013, p. 65-111

Delphine Roy, «Le travail domestique : 60 milliards d'heures en 2010 », INSEE Première, $\mathrm{n}^{\circ}$ 1423, 2012

Mona Zegaï, «La mise en scène de la différence des sexes dans les jouets et leurs espaces de commercialisation », Cahiers du Genre, vol. 49, n 2, 2010, p. 35-54 